\title{
Characterization of dislocations in anisotropic materials by large angle convergent beam electron diffraction
}

\section{J.P. MORNIROLI and P. VERMAUT}

Laboratoire de Métallurgie Physique, Associé CNRS URA 234, Université de Lille I, Bât. C6, 59655 Villeneuve d'Ascq cedex, France

\section{ABSTRACT}

It is shown that the characterization of the Burgers vector of dislocations, by large angle convergent beam electron diffraction, using the cherns and preston rules, is possible in elastically anisotropic materials, provided some care is taken in the choice of the Bragg lines used for the analysis.

\section{INTRODUCTION}

The determination of the Burgers vector of dislocations by conventional transmission electron microscopy is delicate in elastically anisotropic materials because the two invisibility criteria $g \cdot b=0$ and $g \cdot(b \wedge u)$ cannot usually be applied. It has recently been shown that Large Angle convergent Beam Electron Diffraction (LACBED) constitutes a very efficient method of dislocation characterization since the dislocation strain fields produce typical fringes at the intersections with excess or defect Bragg lines which are present on LACBED patterns. The number of interfringes, $n$, is simply equated to the dot product g.b, as indicated by the cherns and Preston rules [1].

- g being a reciprocal lattice vector corresponding to a plane (hkl)

$-b$ being the Burgers vector of the dislocation.

To test the validity of the LACBED method with anisotropic materials, the $\beta^{\prime}$ brass phase was chosen because it exhibits a very high anisotropy factor $2 \mathrm{C}_{44}\left(\mathrm{C}_{11}-\mathrm{C}_{12}\right)=18.7$.

\section{IT. EXPERIMENTAL METHODS}

The cuzn $\beta^{\prime}$ brass phase is an ordered solid solution with structure type B2, CsCl (Fig. 1).

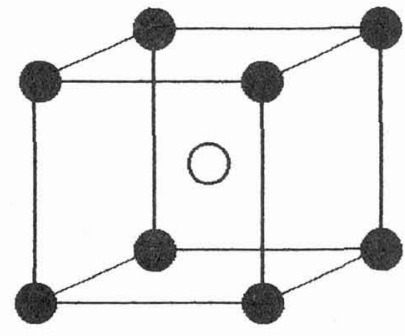

Figure 1.

The $\mathrm{CsCl}$ or $\mathrm{B} 2$ type structure of $\beta^{\prime}$ brass solid solution

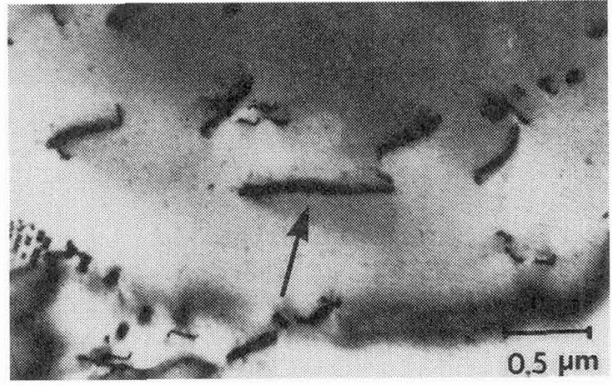

Figure 2

Electron micrograph of the $\beta^{\prime}$ brass specimen 
The $\beta^{\prime}$ specimens examined in this study are composed of large crystals with an average size of about $1 \mathrm{~mm}$. They contain numerous dislocations as shown in Fig. 2 .

Thin foils were prepared by electrolytic polishing using the following solution: $\mathrm{H}_{3} \mathrm{PO}_{4}, \mathrm{C}_{2} \mathrm{H} 5 \mathrm{OH}, \mathrm{H}_{2} \mathrm{O} ; 6: 3: 2$.

These were observed with a Philips CM30 electron microscope working at $300 \mathrm{kV}$ in the nanoprobe mode with a spot size of about $20 \mathrm{~nm}$ and a beam convergence semi-angle of about $3^{\circ}$.

The identification of the Burgers vector is made by means of the Cherns and Preston rules which are summarized in Fig. 3.

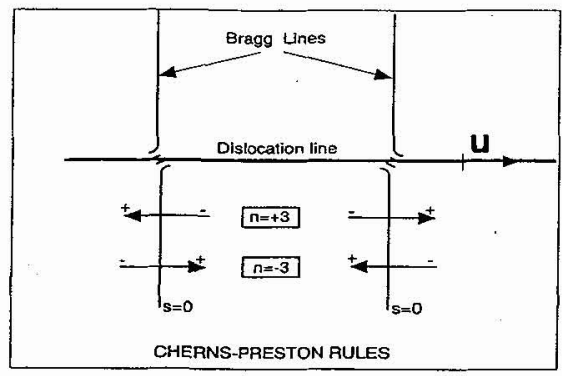

Figure 3

The Cherns and Preston rules

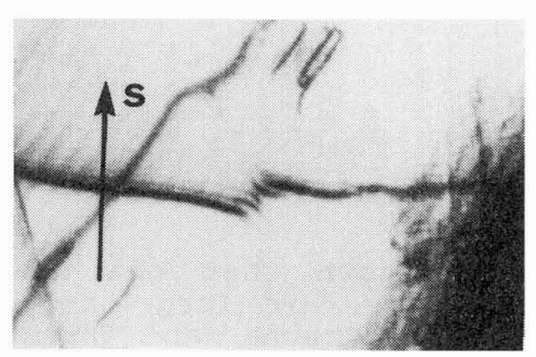

Figure 4

Effect of the dislocation line on $g=501$ giving $n=+4$

This method requires knowledge of:

- $n$, the number of interfringes which are produced at the intersection between the dislocation line and the Bragg line,

- the direction of the dislocation line caracterized by the unit vector $u$ (in agreement with the FS/RH convention for the Burgers circuit),

- the hkl indices of the g Bragg line,

- the direction of the deviation parameter $s$.

These last two features can be identified on an experimental pattern by comparison with theoretical patterns. These may be drawn by means of a computer program which was specially developed for this application and is based on the kinematical theory.

Depending on the dislocation line length, two methods can be used to identify $b$ :

- if the dislocation line is long, then it is possible to place it with respect to the Bragg lines so that it crosses at least simultaneously three different Bragg lines.

- if the dislocation line is short, as it is the case with most of the dislocations present in the $\beta^{\prime}$ brass specimens, the line is successively placed on three Bragg lines. This second method is used in this study.

In both cases, the three corresponding ( $h k l)$ planes chosen should not belong to the same zone axis if ambiguities are to be avoided.

\section{EXPERIMENTAL RESULTS}

The results given here concern the dislocation which is arrowed in Fig. 2 .

The specimen was initially orientated close to the [001] zone axis and the dislocation was successively placed on 14 different hko Bragg lines. The complete results are shown on Fig 5 .

From this Figure the following comments can be made:

The dislocation line exhibits various orientations with respect to the hko Bragg lines examined. It is clearly very difficult to count the number of interfringes, $n$, when the dislocation line is approximately parallel to a Bragg line. 

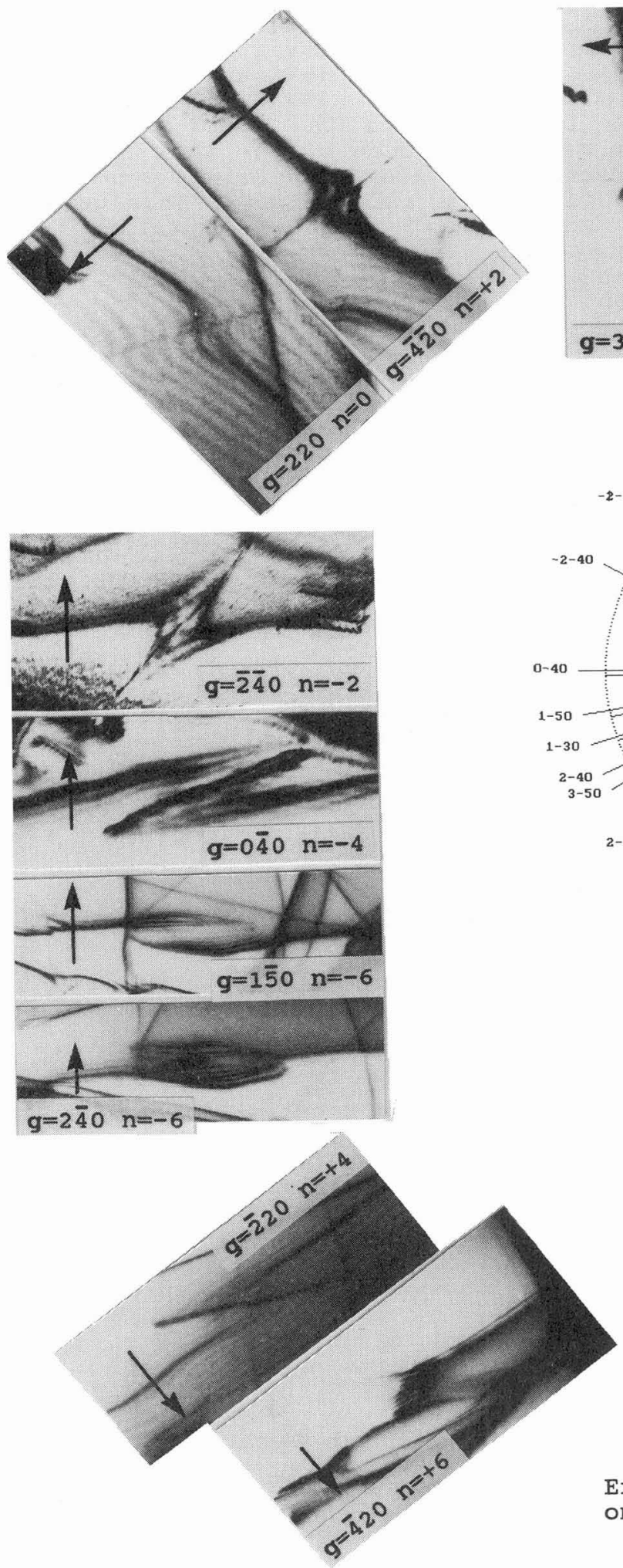
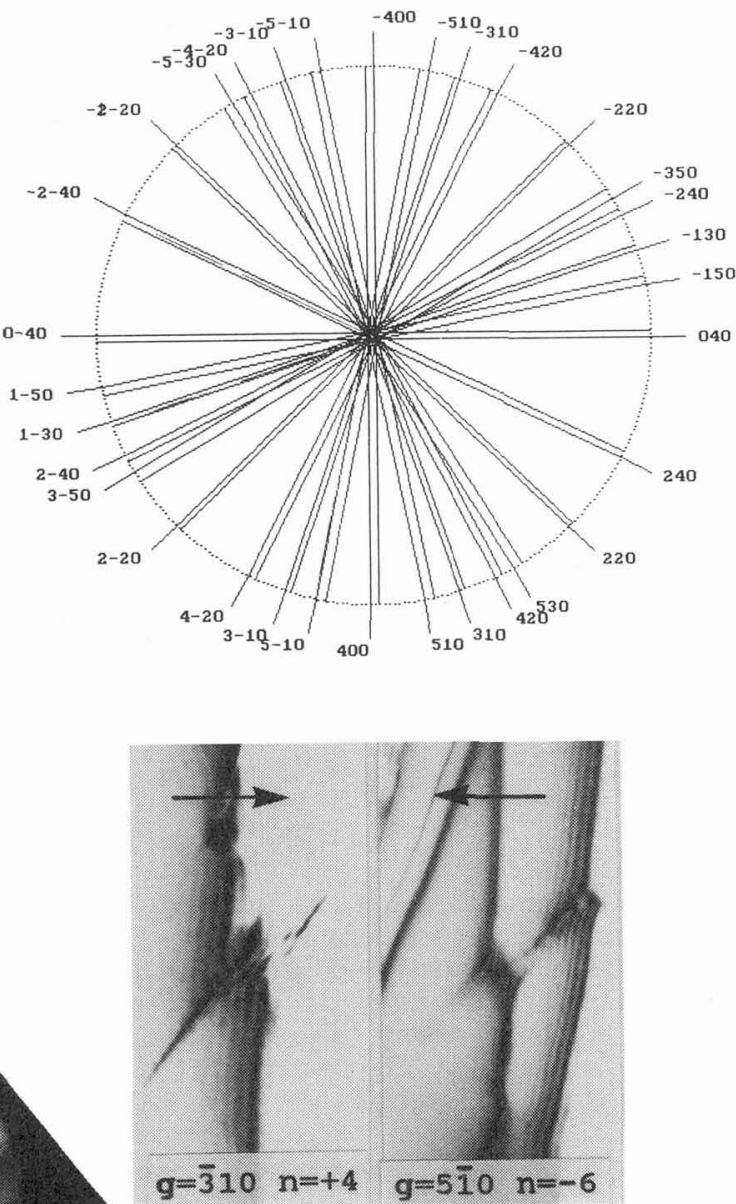

Figure 5

Effect of the dislocation line on various hko Bragg lines 
Only a few Bragg lines give a clear effect from which the number of splittings can be identified conclusively. These were:

$g_{1}=400$ with $n=-4, g_{2}=510$ with $n=-4, g_{3}=5 \overline{1}_{0}$ with $n=-6$ and $g_{4}=\overline{4} 20$ with $\mathrm{n}=+6$.

Since these four Bragg lines correspond to the same zone axis, another experiment was needed in order to deduce $b$. To that aim, the intersection between the dislocation line and Bragg line gs $=\overline{5} 01$ was chosen, which is present on the [115] zone axis (Fig.4). This gave a clear effect with $n=+4$.

By solving the three linear equations:

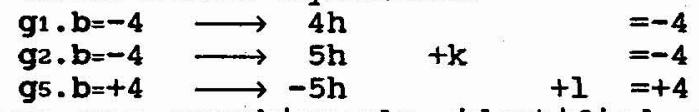

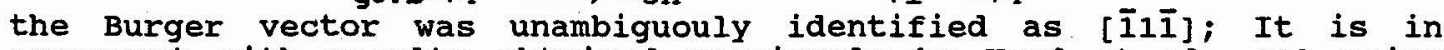
agreement with results obtained previously by Head et al, [2] using computed electron micrographs and by crawford et al. [3].

Most of the splittings exhibit a rather complicated aspect (see especially Bragg contours $g=\overline{4} \overline{2} 0$ and $g=\overline{2} \overline{4} 0$ ). This effect is thought to be connected with anisotropy since we verified that it does not occurs in near isotropic materials (silicon for example).

The Bragg contour $g=220$ exhibits an abnormal aspect despite its value $\mathrm{n}=0$. Some splittings are observed, which initially seem to have a normal aspect but then lead to erroneous values for $n$. Using the example given in Fig.6., which corresponds to the 400 Bragg contour with $n=4$, the distinction between $n=4$ and $n=5$ is not made evident.

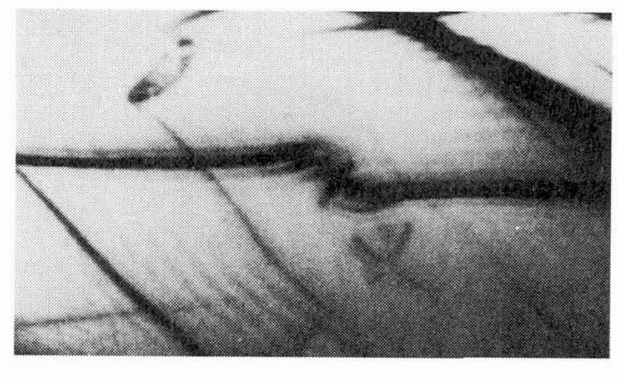

Figure 6

$g=400$ Bragg contour with $n=+4$.

IV. CONCLUSIONS

The dislocations present in this anisotropic material give more or less complicated splittings at the intersections between the dislocation lines and the Bragg contours present on LACBED patterns. Some splittings are of normal appearence and verify the cherns and Preston rules; many have a complex aspect which is probably connected with anisotropy. It is therefore possible to characterize dislocations in anisotropic materials by LACBED provided the Bragg lines used for the analysis are carefully chosen. Any line which exhibits an abnormal aspect should be discarded.

[1] CHERNS D. and PRESTON A.R., J. Electr. Microsc. Techn. 13, (1989), 111.

[2] HEAD A.K., HUMBLE P., CLAREBROUGH L.M., MORTON A.J. and FORWOOD C.T. Computed electron micrographs and defect identification, North Holland Publishing Company, Amsterdam 1973.

[3] CRAWFORD R.C. and MILLER D.R., Phil. Mag. 1966,8, 1071. 